\title{
Simulation of Construction Disturbances with Agent-Based Petri Nets and Employing a BIM in Expert Building Time Analyses
}

\author{
Ralf Gnerlich ${ }^{1 *}$, Antje Tiesler ${ }^{1}$, Volkhard Franz ${ }^{2}$ \\ ${ }^{1}$ FairCM² GmbH, Freie-Vogel-Straße 393, 44269 Dortmund, Germany; *gnerlich@fcm2.de \\ 2University of Kassel, Institute of Construction Management, Mönchebergstraße 7, 34125 Kassel, Germany
}

SNE 30(4), 2020, 203-212, DOI: 10.11128/sne.30.tn.10542 Received: July 13, 2020 (Selected ASIM SPL 2019 Postconf. Publ.); Revised: Oct. 11, 2020; Accepted: Oct. 20, 2020

SNE - Simulation Notes Europe, ARGESIM Publisher Vienna ISSN Print 2305-9974, Online 2306-0271, www.sne-journal.org

Abstract. This paper is based on a research project focused on updating a disturbance-modified schedule for the evaluation of building claims. In this context, the quantification of time effects in expert analyses is discussed. This novel approach is based on a time-discrete simulation method using an agent-based simulation with Petri nets and building information models. Here, the focus is on the reconfiguration of the composition structure of the Petri net, which is triggered by events in the sequence structure. In addition, this paper presents a conceptual proposal how to chronologically integrate documents into the Petri net structure. Thus, the simulation model and the building claims can be verified and validated. Specialized transitions are also used to implement document integration into the Petri net model.

\section{Introduction}

Forensic expert reports on claims due to disruptions in the construction process are often not recognized by German courts. The reasons for this can usually be traced back to an impracticable presentation of the facts in expert reports [1]. Tiesler [1] has shown that experts often fail to conclusively identify and explain the necessary causal relationships in the disrupted construction process.

In this context, there is no general approach to solve such issues in German expert practice. Rather, individual case-related approaches are used to explain the disrupted construction process in the expert reports.
Within this setting, the expert analysis must be adapted to the respective problem case. Therefore, the choice of a suitable methodology for carrying out the analysis always depends on the individual case studies.

One challenge in the forensic analysis of construction time is that disrupted construction processes can be extremely complex systems. Complexity analyses are, therefore, essential to meaningfully separating and understanding the effects of disturbances during the construction time under consideration.

German expert practice currently mainly uses project management software, which is usually based on the critical path method (CPM), to perform complex analyses of disturbed construction processes [1-3]. However, such trivial calculation methods can lead to inconclusive results [2-5].

\section{Literature Review}

Experts rarely ask whether CPM is suitable for the conclusive analysis of complex construction time disturbances. Instead, they scrutinize the methods of detection for target-actual comparisons used rather than the calculation methods of valid process durations [1].

Modern approaches solve this challenge with building information models (BIMs) linked to CPM networks. [6]. Such procedures create so-called four-dimensional (4D) models or 4D BIMs (i.e., 3D geometry + 1D time). For 4D BIMs, it is claimed that the validity of the construction time analysis is stronger [7].

To obtain knowledge using the $4 \mathrm{D}$ model, the $3 \mathrm{D}$ components of the BIM must be played back as a $4 \mathrm{D}$ visualization using time information. The modelled dependencies of the production processes in particular are then clearly illustrated. 
Overall, a 4D model can present more information than a bar chart and is more comprehensible for third parties. Similarly, a 4D model provides a greater understanding during the modelling phase.

This approach seems to have many advantages compared to conventional presentation methods. Thus, in the past, researchers discussed these advantages for forensic construction time analyses [8-10].

\subsection{Research Gap}

4D visualizations are helpful in clarifying human perceptions [11]. Moreover, it has been shown that manually performed construction time analyses can produce morerealistic results if calculations are based on BIMs [18].

However, the logic and practicability of the calculated processes in such methods are still based on the subjective, transcendent perception of the modeler. In addition, forensic analyses of construction time disturbances often require the objective consideration of the many complex environmental influences on a building project [3]. Here, it is possible that the circumstances substantiating the claim were not thought through holistically. For example, the dependencies of complex design processes cannot be recognized or misunderstood [3]. It follows that in many cases no practicable system behavior can be assumed due to the limitations of human perception [3, 4].

For this reason, despite 4D support, invalid expert opinions can still emerge. This can be especially true if the time sequences are modeled using CPM [3]. Regarding justiciable claims relating to disruptions to construction processes, gaps may arise in the argumentation line $[1,3]$. Inconsistent calculation results can be challenged in court easily. In such cases there is a risk that a court will declare the expert report to be unsuitable and entitled claims are lost.

The challenges discussed above are well-known simulation topics. This raises the question of whether simulation techniques in combination with BIMs can be used to prove claims due to construction process disturbances.

Construction process simulations have the advantage that they can execute large-scale calculations. This usually enables more intensive and realistic analyses of complex processes [21-23]. For example, complex spacetime conflicts can be considered due to the realistic building geometry [24, 25]. In addition, virtual production processes can be interrupted, delayed, inhibited, or accelerated with specific parameter inputs [21, 23].
These properties of computer-aided simulations are suitable for disturbance-modified schedule updates and the evaluation of entitled claims [3, 13, 14, 23], which can lead to unexpected analysis results. The anticipation that unexpected results will be simulated can lead to a greater objectivity in the analysis process, because the simulation user will inevitably examine the simulation results before they can be understood [3].

Indeed, only a few researchers consider using BIMbased simulation methods to solve these challenges [1219]. However, there are almost no concrete proposals for solutions regarding a suitable calculation methodology (some exceptions are [3, 20]).

\subsection{Further Procedure}

This paper proposes an approach using a simulation method for practicable production processes to provide expert reports about construction time claims. In this context, Gnerlich's approach [3] is summarized. For this purpose, this paper first discusses the related simulation methods in the BIM context. Subsequently, the simulation design follows. Finally, the paper concludes with an outlook.

Overall, the conceptual design and less technical details are dealt with. Mathematical descriptions are also omitted from this article. The agent logic is explained in more detail by Gnerlich [3].

\section{Related Simulation Methods}

Gnerlich's simulation approach comprises an agentbased Petri net using a BIM to simulate disturbance-modifying effects for an objective schedule update. The method also includes a mechanism for chronological management of external documents. This linkage is suitable for model verification and validation as well as for the legal presentation of entitled claims. All aspects are realized by using a Petri net as a simulator.

\subsection{Benefits of Petri Net Simulation}

Token-based semantics are regularly used for executable BPMNs, EPKs, and UML displays [26] or for executing software and hardware $[27,28]$ due to their causal computational logic. Accordingly, Samkari [29] showed that extremely fast and extensive background calculations of BIM-based construction process simulations can be realized with Petri nets. 
He also demonstrated that the simulation modelling can be done directly in the BIM viewer. In this case, the calculation of the sequence structure of the Petri net is realized by an algorithm in the application's background. For this purpose, subject-specific user interfaces can be designed for intuitive input [3]. Users who are not simulation experts can, thus, contribute their technical knowledge more easily and quickly $[29,30]$. This can be particularly helpful for updating a disturbance-modified petri net model, because a manual modelling can become extremely complicated and error-prone due to the increased complexity [29].

Petri nets are suitable for simulating complex and causal relationships of user-defined model processes [3, $27,31]$. In addition, Petri nets can represent a composition structure in ordered detail $[3,27,31]$; thus, it is possible to define modular subnets in several hierarchical levels [27, 31, 32]. For example, these properties allow for dividing the system "construction project" into its components [3, 31, 32]. Based on this procedure, the simulation results can later be structured meaningfully. In addition, such a composition structure usually contributes to a certain clarity and readability of the simulation results [32].

Beyond that, the Petri net markings represent reproducible intermediate states of the simulation runs [3]. Using these markings, the various calculation steps can later be traced back in detail $[3,29]$, which enables previously executed simulation runs to be rewound, the model design revised, and the simulation run restarted, as necessary.

\subsection{Benefits of BIM- and Agent-based Simulation}

Some simulation researchers have demonstrated that users gain a better understanding of production processes when agent-based simulation models use BIMs as spatial model environment $[22,30]$. Agent-based simulation can reproduce an emergent model behavior corresponding to more-realistic system behaviors [22, 30]. An example of emergence results from changes in the construction process as a result of disturbances. Emergence often stems from a changed behavior of the ancillary trades. Those effects are core questions that often must be clarified in expert reports [14, 33].

In addition, BIMs enable a component-related and chronological integration of external documents about the construction project $[3,16,34,35]$.
This is particularly suitable for the analysis of disrupted construction processes. Thus, the accuracy of the simulation model can be checked and a claim can also be verified [3].

\subsection{Useful Petri Net Concepts}

Damrianant and Wakefield [36] present switch functions for delays and interruptions to be able to simulate disturbances with (cyclic) Petri nets. For this purpose, they combined a Petri net with additional modelling elements for switching delay factors on and off. They also simulated interruptions by temporarily ignoring the tokenized resources in the process calculations.

Further approaches can be found in so-called "time Petri nets", which hold the tokens in the transitions or places and, thus, delay the switching (e.g., [37]). In contrast, Samkari [29] presents a simulation approach with agent-based Petri nets.

In this case, the agents are an additional constraint for switching the transitions. Here, the process calculation of the transitions uses a guard function, which checks the availability of the necessary resources before switching. An activated transition only switches completely if all required agents are available [29]. The Petri net is also constructed as a predicate transition net. Each place has only one input and one output edge, like a marked graph. Each newly created token represents an elementary model state. According to this, Samkari [29] dispensed with any cyclical structures.

In contrast to time Petri nets, the simulation time is not stopped at so-called "timed Petri nets" [38]. Instead, the tokens transmit time information from the preset transitions to the activated transitions. If a transition is switched, all time information is compared and calculated. This method enables very fast simulation runs of complex construction process scenarios [29].

To a large extent, the simulation approach used in this paper was oriented towards Samkari's [29] agent-based Petri net method. In addition, similar switch functions from Damrianant and Wakefield [36] were used to model date discrete changes as construction process disturbances.

\section{Simulation Design}

The basis for agent movements is a path network in the BIM, which consists of path edges and nodes. Figure 1 shows a virtual BIM with such a path network. 


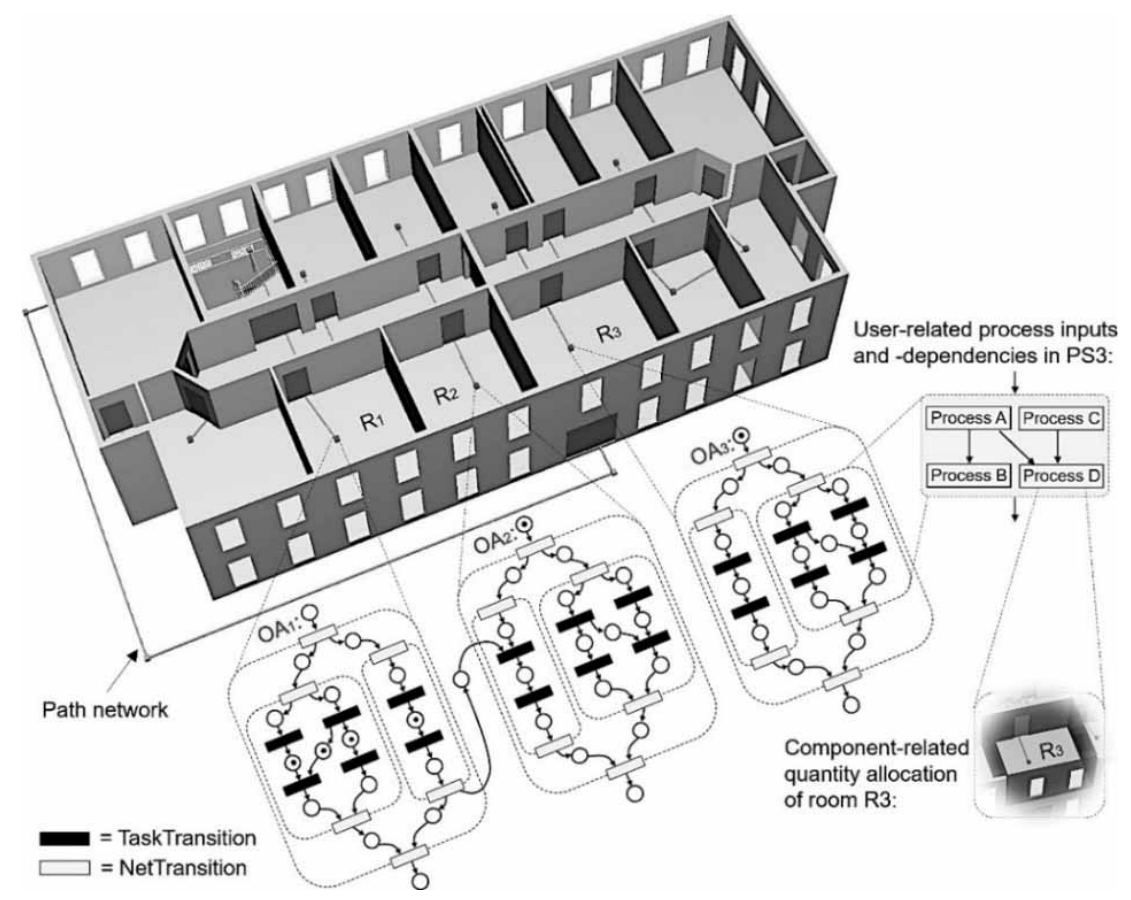

Figure 1: Definition of the local operational areas: $O A_{i}$ are subnetworks.

A dark color indicates the path nodes that are used for the user-related definition of local operational areas (OAs), which can refer to user-defined rooms, floors, or sections, etc., in the BIM.

Figure 1 indicates that the Petri net is derived from the process inputs and quantity assignments of the BIM. Thus, the user does not need to model a Petri net structure. According to Figure 1, all local OAs can be converted into executable Petri net structures. The Petri net is, therefore, the interface between the BIM and the Petri net. The Petri net itself is in the background of the simulation application as the "calculation tool" in the sequence structure and is invisible to the user. However, it is possible for the user to access the local OAs to carry out the simulation modelling there.

The right side of Figure 1 shows an example section that illustrates the user-specific process definitions and process dependency entries using the local OAs. In these, it is possible to model individual composition structures, e.g., to delimit the (sub)processes of individual construction trades from each other. The process structures are defined by entering simple arrows. They specify the necessary dependencies of sequential processes. Overall, dependencies between the local OAs can also be defined.

For the calculation of the construction process durations, the entered processes must be offset against the respective quantities of components in the BIM.
To do this, the user can get the properties of the topologically arranged building objects of the BIM. There are no conditions for when the user chooses the component objects. With this method, the local OAs can include processes that apply to several floors or sections of any size.

However, the user should consider the spatial proximity of the local OAs inserted in the BIM, which can be important later to better understand the simulated production processes.

To finally simulate the construction production processes, it may be necessary to assign resources to the processes, such as worker groups, equipment, dates and deadlines, materials, or standing areas of the local OAs. However, no resource allocation is necessary for curing or drying processes. All inputs necessary for the calculation of the process duration have now been described.

During simulation execution, the workers (agents) must get to the local OAs using the path network and fulfill the defined work tasks. To ensure that a production sequence is followed as far as possible, the user can define one or more production direction(s) for the worker groups using the local OAs. The production directions can be prioritized, so that the workers (agents) in a group can give preference to specific production processes in case of alternative measures. 


\subsection{Petri Net Components}

Subnetworks are derived from the composition structure of the local OAs of hierarchically combined processes. These are limited within the Petri net structure by two NetTransitions. One NetTransition forms the input and the other NetTransition forms the output of each subnet. In between are the elementary TaskTransitions, which are derived from the entered processes and execute the task calculations (according to Figure 1).

As soon as a TaskTransition switches, all associated building-component-related quantities and associated trade-specific process definitions are offset against each other. A TaskTransition can only be switched if its preset is activated and the resources required for the process are available [3]. For example, enough material must be available for switching resource-dependent TaskTransitions. In addition, a permitted number of agents must be able to reach the local OAs using the path network.

For the calculation of the process duration, a time effort value for labor productivity must be specified in the process definition. The time effort value can be weighted by various parametric effort factors.
Depending on the value, these expense factors offer several possibilities. The simulated production processes can be inhibited, accelerated, or interrupted. In the expert analyses of construction time, statements on necessary productivity as a result of experimental weighting combinations can, thus, be obtained. From the weighted time expenditure value, findings of the simulated working speeds can be derived. Figure 2 provides an overview of the possible weightings and the formula for the process duration calculation. This is processed by default when switching a TaskTransition.

For the simulation of discrete time construction process disturbances, the Petri net is divided into two subnetworks. In terms of Figure 3, these are the ProcessBranchnet and the Date-Time-Branchnet. The subnetworks switch alternately during a simulation run and mutually renew their previous Petri net structure.

Based on Figure 1, the Process-Branchnet includes all subnetworks of the local OAs. Figure 3 illustrates how the subnetworks can be organized into a hierarchical composition structure. In this way, e.g., different companies can be separated from each other with their trade and service descriptions down to the elementary work tasks.
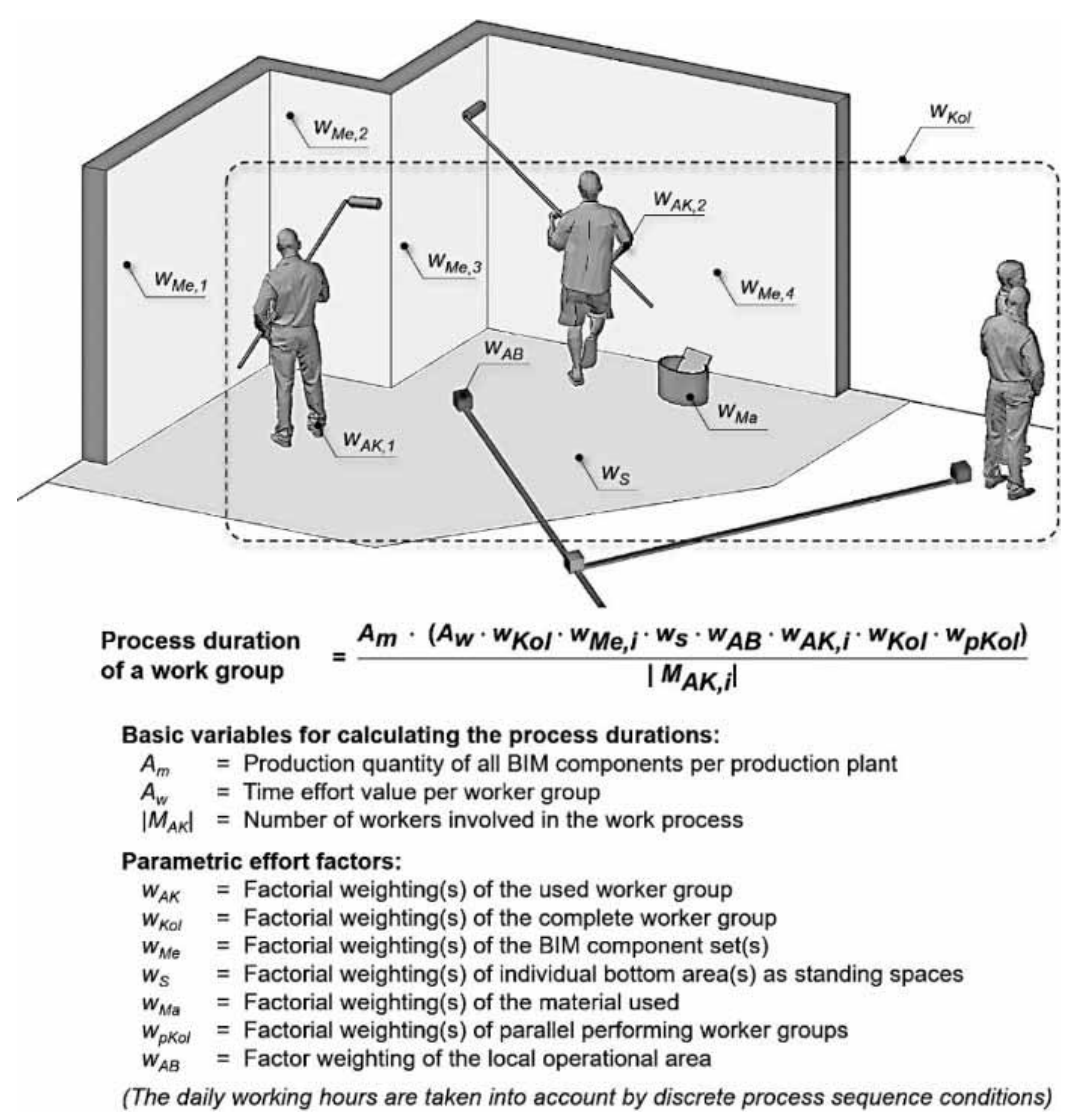

Figure 2: Calculation content of a TaskTransition [3]. 


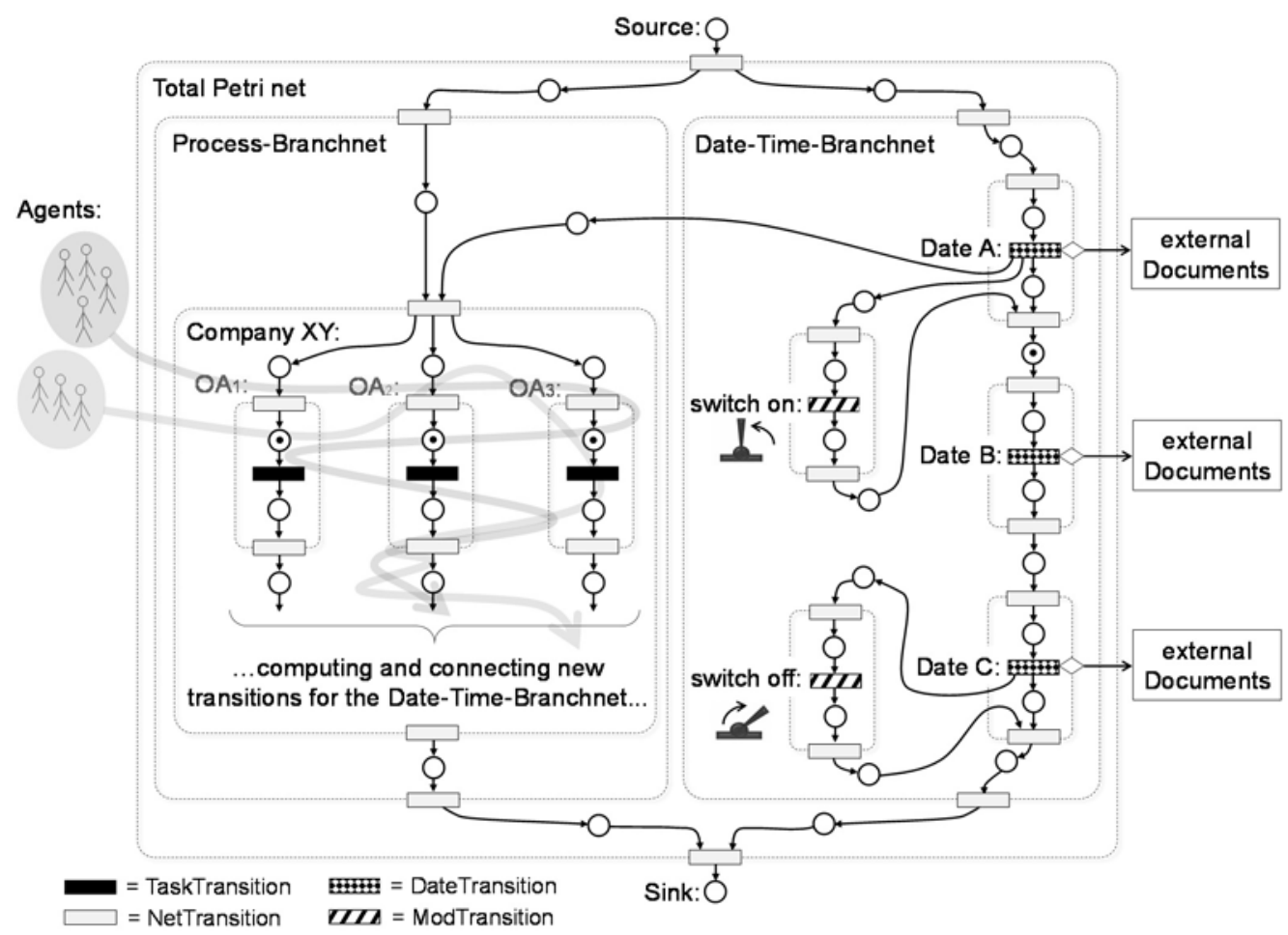

Figure 3: Overview of the entire Petri net structure.

Work tasks are represented by TaskTransitions, which calculate the process times for start, intermediate, and end dates when they are switching tasks. During a simulation run, all dates calculated by the ProcessBranchnet are successively transferred to the Date-TimeBranchnet as date-bound DateTransitions. Afterwards, they are linked in a chronological order.

DateTransitions are time discrete starting conditions for any number of TaskTransitions. The user can also integrate DateTransitions into the Petri net as time-critical process conditions if dates are entered and linked to the processes. External attached documents can also be assigned to these dates or the resulting DateTransitions. This enables the validation and verification of the simulated process sequences by means of comparison values that are fixed for specific dates.

Figure 3 shows that DateTransitions and ModTransitions are in the Date-Time-Branchnet. ModTransitions perform switching functions for time-discrete disturbances in the Process-Branchnet. Therefore, they must be arranged within the Petri net directly behind a DateTransition and change their discrete values during switching. For example, when switching ModTransitions, any number of agents can be added or removed from the simulation model.
In this way, the working hours or absences due to illness of the employees can be represented. With this principle, any component objects of the BIM can also be blocked so that further work in certain local OAs is no longer permitted. Furthermore, it is possible to renew discrete model values during a simulation run with isolated ModTransitions. For example, changes of the displayed effort factors are achieved according to Figure 2. Figure 3 illustrates the principle of extraction and addition using "switch on/off" symbols. For clear differentiations in the Date-Time-Branchnet, the DateTransitions and the ModTransitions are enclosed by their own subnets.

\subsection{Structural Petri Net Update}

Figure 4 shows six steps of mutual switching for the update of a disturbance-modified schedule of the Petri net. Mutual switching means that one subnetwork must always wait while the other subnetwork switches.

Overall, the six steps illustrate the markings of the Petri net states that are reached during model execution. Furthermore, the waiting states of the subnets are also shown.

For example, the right side of Figure 4 shows the maximum state descriptions that the Process-Branchnet can assume until the Date-Time-Branchnet is switched. 


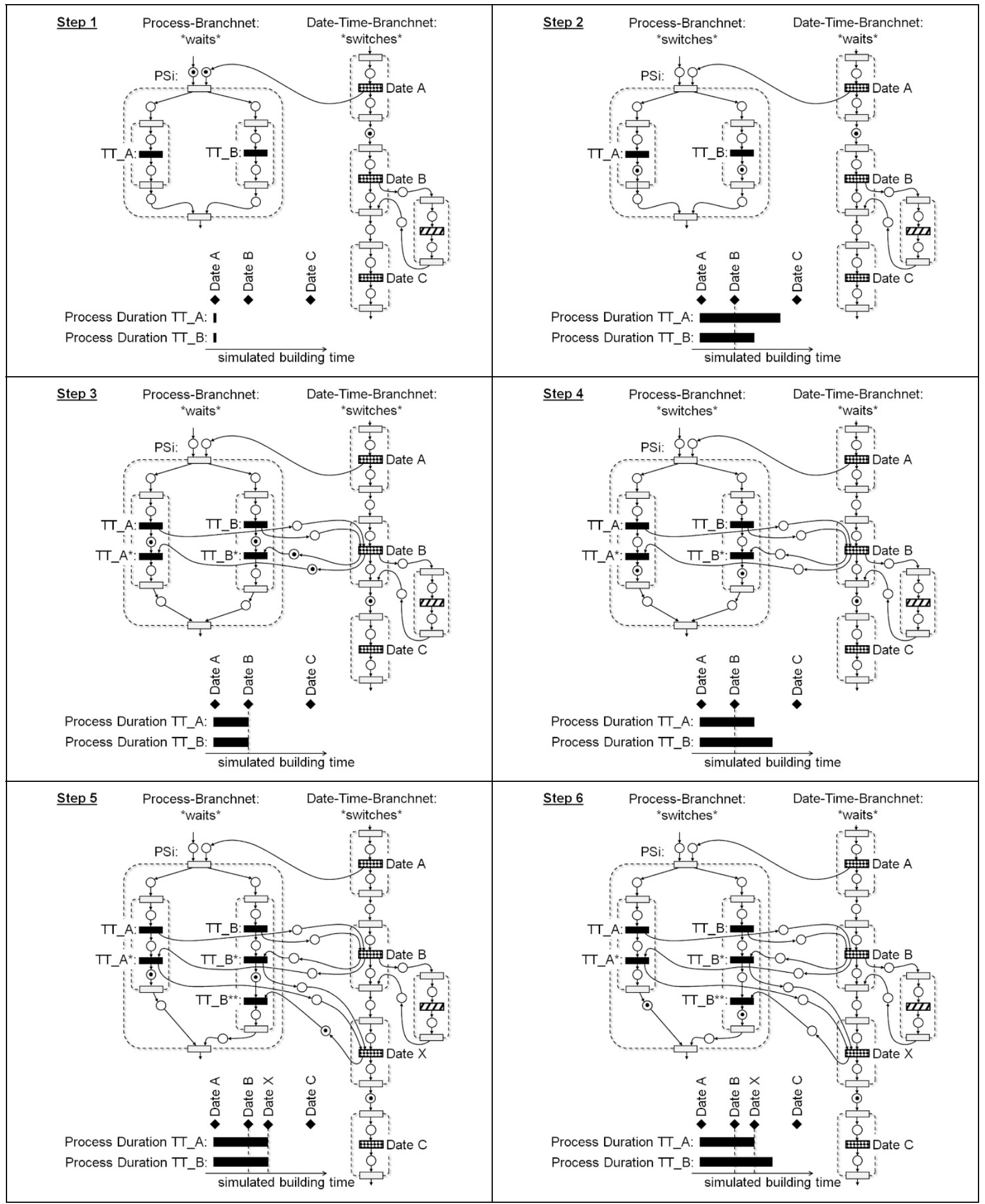

Figure 4: Mutual switching principle in six steps. 
In contrast, the left side shows the switched DateTime-Branchnet. A bar chart is displayed for each step. There, the black bars show the calculated process durations. Black diamonds represent dates in the Date-TimeBranchnet.

In Step 1 all transitions of the Date-TimeBranchnet are switched until a token is between the two subnets of Date $A$ and Date B. Afterwards the switching of the two TaskTransitions TT_A and $T T_{-} B$ in the ProcessBranchnet follows in Step 2. The resulting tokens remain in the direct downstream area of the two TaskTransitions. Here, the lower bar chart shows that both TaskDurations have overlapped the Date $B$ of the DateTransition.

For this reason, Date $B$ remains the next earliest date. All overlapping process durations are cut and removed. Therefore, the simulator copies and links the two TaskTransitions according to Step 3.

In this case, the TaskTransition $T T_{-} A^{*}$ is a copy of TT_A. All copied TaskTransitions share the process definitions of their original TaskTransitions. However, they differ in the calculated performance states. Thus, from the original TaskTransition TT_A, the previously calculated process state up to the time of the intersecting date remains as the final state. On the other hand, the copied TaskTransition $T T \_A *$ receives the last reached process state as an initial state. In addition, all affected TaskTransitions are linked to the DateTransition of Date B according to Step 3. For that, it is necessary to create new tokens, which pass the calculated performance states to the following transitions.

In Step 4, the ModTransition also switches, which means that the process duration of TT_B* is longer than of $T T \_A^{*}$. The transitions in the Date-Time-Branchnet switch until a token is between the subnets of Date $B$ and Date $C$. Step 5 shows the situation that the calculated date (Date $X$ ) is the next earliest compared to Step 3. Analogous to Step 3, the simulator copies and links all TaskTransitions whose process durations overlap Date X. For this purpose, a new subnetwork is automatically created by the simulator for Date $X$ in the Date-Time-Branchnet. For the sake of completeness, Step 6 shows the switching process that determines the final process durations.

During the structural Petri net update, the construction production processes are shifted forward or backward in time. The documents that are stored in the Petri net remain unchanged at the specified dates of the DateTransitions.
Their contents describe those model states that must be reproduced by simulation experiments as a result of the structural Petri net update.

On the one hand, a sufficiently justified verification is present if the state descriptions of the documents correlate with the simulation results in time. On the other hand, the simulation results can be validated with the simulated process sequence behavior. In such cases, experts must estimate whether the construction processes were practicable and under which parameter settings the step-by-step verifications were possible. The effort factors can be combined to determine deviations or to make other statements about the building time analysis, e.g., regarding missing information. Further details can be found in [3].

With the automated update rules presented here, a Petri net can be used as a background simulator. Despite reconfigurations, an executable process sequence structure can be realized. For example, the user can already be informed during his or her input whether the process sequence structures can be executed or not. In summary, the presented simulation principle can generate a very complicated process sequence structure with extremely complex construction processes.

\section{Summary and Perspectives}

This paper presented an approach to simulate construction process disturbances with agent-based Petri nets using a BIM for expert construction time analyses.

In contrast to the CPM technique, which is predominantly used in current consultant practice, the benefit of the approach presented here is the availability of chronological and process-related input options, in order to achieve disturbance-modifying effects for an objective schedule update

With the reconfigurable properties of the designed Petri net, complex simulation experiments are possible, which can well reproduce an emergent system behavior. The integration of external documents provides clues for validation and verification of the model behavior. Expert opinion argumentation lines can also benefit from this Petri net, because the simulated performance progress can be compared with the attached documents.

Experiments have already been carried out to simulate complex construction sequences of disturbed production processes. Gnerlich's [3] research project presents several simulation runs that illustrate the functionality of the approach presented in this paper. 


\section{References}

[1] Tiesler A. Entwicklung eines substantiierten Kausalitätsnachweises von Ursache und Wirkung für Bauablaufstörungen auf Basis der deutschen Rechtsprechung. Institute of Construction Management. Kassel: University of Kassel; 2018.

[2] Eschenbruch K, von Rinteln C. Bauablaufstörung und Terminfortschreibung nach der VOB/B - Stresstest für die baubetrieblichen Gutachten. NZBau; 7/2010: 401-411.

[3] Gnerlich R. Entwicklung eines Konzepts zur digitalen Untersuchung von Bauzeitverzögerungen auf Grundlage einer BIM-basierten Bauablaufsimulation. Institute of Construction Management. Kassel: University of Kassel; 2019. doi: 10.19211/KUP9783737606837

[4] Eden C, Williams T, Ackermann F. Analysing project cost overruns: Comparing the "measured mile" analysis and system dynamics modelling. International Journal of Project Management. 2000; 23(2): 135-139. doi:10.1016/j.ijproman.2004.07.006

[5] Drittler M. Nachträge und Nachtragsprüfung beim Bauund Anlagenbauvertrag, Lösungen zum Erkennen, Sichern, Begründen, Nachweisen, Prüfen von Ansprüchen aus Auftragnehmer- und Auftraggeberinteresse. $3^{\text {rd }}$ ed. Köln: Werner; 2017, 800 pages.

[6] D'Amico F, D'Ascanio L, De Falco MC, Presta D, Tosti F. BIM for infrastructure: An efficient process to achieve 4D e 5D digital dimensions. AIIT $2^{\text {nd }}$ International Congress on Transport Infrastructure and Systems in a Changing World, 2019 September; Rome, Italy.

[7] Koo B, Fischer M. Feasibility study of 4D CAD in commercial construction. Journal of Construction Engineering and Management. 2000; 126(4): 251-260. doi: 10.1061/(ASCE)0733-9364(2000)126:4(251)

[8] Wotschke P, Kindermann G. Die Simulation des (un)gestörten Bauablaufs als Beweismittel in einem Streitfall. In: Simulation von Unikatprozessen: Neue Anwendungen aus Forschung und Praxis, III Tagungen und Berichte. 2011 March; Kassel, Germany. 93-107.

[9] Guévremont M, Hammad A. Visualization of delay claim analysis using 4D simulation. Journal of Legal Affairs and Dispute Resolution in Engineering and Construction. 2018; 10(3): 05018002. doi: 10.1061/(ASCE)LA.1943-4170.0000267

[10] Ali B, Zahoor H, Nasir AR, Maqsoom A, Khan RWA, Mazher KM. BIM-based claims management system: A centralized information repository for extension of time claims. Automation in Construction. 2020; 110, 102937. doi: 10.1016/j.autcon.2019.102937
[11] Franz V, Samkari K, Gnerlich R. Perception of a building construction schedule. EAIA and MatH '13: Proceedings of the Emerging M\&S Applications in Industry \& Academia / Modeling and Humanities Symposium. 2013 April; San Diego, California, United States.

[12] Koc S, Skaik S. Disputes Resolution: Can BIM help overcome barriers? CIB 2014: Proceedings of the 2014 International Conference on Construction in a Changing World. 2014 May; Dambulla, Sri Lanka.

[13] Valavanoglou A, Heck D. Building information modeling and forensic analysis of delay and disruption. In: Komurlu R, Gurgun AP, Singh A, Yazdani S, editors. Interaction between Theory and Practice in Civil Engineering and Construction. 2016; 527-532. doi: 10.14455/ISEC.res.2016.50

[14] SCL-Protocol. Protocol of Delay and Disruption Protocol $2^{\text {nd }}$ Edition. Hinckley, UK: SCL; 2017. 85 pages.

[15] Gnerlich R. BIM und Simulation - Erste Ansätze und Eingrenzungen für baubetriebliche Gutachten über gestörte Bauabläufe im deutschen Baurecht. 28. BBB-Assistententreffen. 2017 May; Kaiserslautern, Germany, 75-89.

[16] Soltani Z, Anderson S, Kang J. The challenges of using BIM in construction dispute resolution process. $53^{\text {rd }}$ Annual International Conference of the Associated Schools of Construction. 2017 April; Seattle, DC. 771-776.

[17] Chou HY, Yang JB. Preliminary evaluation of BIMbased approaches for schedule delay analysis. IOP Conference Series: Materials Science and Engineering, 2017 June; Prague, Czech Republic; 245(6): 062048. doi: 10.1088/1757-899X/245/6/062048

[18] Gnerlich R, Tiesler A, Franz V. Einfluss objekt-verteilter Zeitaufwandswerte auf die Anspruchsbewertung gestörter Bauabläufe. BauSIM, 7. Deutsch-Österreichische IBPSA Konferenz. 2018 September; Karlsruhe, Germany. 44-56.

[19] Eschenbruch K, Gerstberger R. Zeitenwende für baubetriebliche Gutachten. Bauwirtschaft (BauW). 2018; 1: 45-56.

[20] Su Y, Isaac S, Lucko G. Integrated temporal-spatial model for construction plans with boolean logic operators. Journal of Construction Engineering and Management. 2018; 144(4): 04018009. doi: 10.1061/(ASCE)CO.1943-7862.0001450

[21] Ben-Alon L, Sacks R. Simulating the behavior of trade crews in construction using agents and building information modeling. Automation in Construction. 2017; 74: 12-27. doi: 10.1016/j.autcon.2016.11.002 
[22] Gnerlich R, Tiesler A, Möhring F, Franz V. Baubetriebliches Statement über Prognosen und Rekonstruktionen mittels BIM-basierter Simulation zur Bewertung gestörter Bauabläufe. Sonderband Digitalisierung anlässlich des 4. BIM-Symposiums Rheinland-Pfalz. 2018 October; Kaiserslautern, Germany. 45-56.

[23] Akinci B, Fischen M, Levitt R, Carlson R. Formalization and automation of time-space conflict analysis. Journal of Computing in Civil Engineering. 2002; 16(2): 124-134. doi: 10.1061/(ASCE)0887-3801(2002)16:2(124)

[24] Mirzaei A, Nasirzadeh F, Parchami Jalal M, Zamani Y. 4D-BIM dynamic t-space conflict detection and quantification system for building construction projects. Journal of Construction Engineering and Management. 2018; 144(7): 04018056. doi: 10.1061/(ASCE)CO.1943-7862.0001504

[25] Van der Alst WM. Geschäftsprozessmodellierung: Die „Killer-Applikation“ für Petrinetze. InformatikSpektrum. 2014; 37(3): 191-198. doi: 10.1007/s00287-013-0756-2

[26] Peterson JL. Petri net theory and the modeling of systems. Englewood Cliffs, NJ: Prentice-Hall; 1981. 288 pages.

[27] Wakefield RR, Sears GA. Petri nets for simulation and modeling of construction systems. Journal of Construction Engineering and Management. 1997; 123(2): 105-112. doi: 10.1061/(ASCE)0733-9364(1997)123:2(105)

[28] Samkari K. Automatisierungsansätze zur Verbesserung der Simulation von Bauabläufen im Hochbau. Institute of Construction Management. Kassel: University of Kassel; 2014.

[29] Kugler M. CAD-integrierte Modellierung von agentenbasierten Simulationsmodellen für die Bauablaufsimulation im Hochbau. Institute of Construction Management. Kassel: University of Kassel; 2012.
[30] Franz V. Planung und Steuerung komplexer Bauprozesse durch Simulation mit modifizierten höheren Petri-Netzen. Kassel: Gesamthochschule Kassel; 1989.

[31] Schopbach H. Ansätze zur Kostensenkung in Konstruktion und Baubetrieb durch Einsatz mathematischer Optimierungsmethoden. Institute of Construction Management. Kassel: University of Kassel; 2001.

[32] BGH. Urteil vom 24.02.2005: Entgangener Gewinn aus nicht durchgeführtem Bauvertrag und Behinderungsschaden, AktZ. VII ZR 225/03. Neue Juristische Wochenschrift (NJW), 2005; (23): 1650-1653.

[33] Valavanoglou A, Heck D, Rebolj D. Construction delay and disruption claims assisted through BIM technology. LC3 2017: Vol. I-Proceedings of the Joint Conference on Computing in Construction (JC3). 2017 July; Heraklion, Greece. 391-398. doi: 10.24928/JC3-2017/0192

[34] Gibbs DJ, Lord W, Emmitt S, Ruikar K Interactive exhibit to assist with understanding project delays. Journal of Legal Affairs and Dispute Resolution in Engineering and Construction. 2017; 9(1): 04516008. doi: 10.1061/(ASCE)LA.1943-4170.0000198

[35] Damriantant J, Wakefield RR. An alternative approach for modeling of interference in discrete-event systems. Civil Engineering and Environmental Systems. 2000; 17(3): 213-235. doi: 10.1080/02630250008970283

[36] Ramchandani C: Analysis of asynchronous concurrent systems by Petri nets. Project MAC, TR-120. Cambridge, MA: MIT; 1974.

[37] Merlin P, Farber D. Recoverability of communication protocols - Implication of a theoretical study. IEEE Transactions on Communications. 1976; 24(9): 10361043. doi: 10.1109/TCOM.1976.1093424. 